\title{
Curcumin improves perfusion recovery in experimental peripheral arterial disease by upregulating microRNA-93 expression
}

\author{
JINFENG ZHANG ${ }^{1,2}$, QIONGTAO WANG ${ }^{1}$, GUOTAO RAO $^{1}$, JUNYING QIU ${ }^{1}$ and RONGHUA HE ${ }^{1}$ \\ ${ }^{1}$ Department of Cardiology, The Central Hospital of Xiaogan, Wuhan University of Science and Technology, \\ Xiaogan, Hubei 432000; ${ }^{2}$ Department of Cardiology, Cardiovascular Research Institute, Wuhan University, \\ Hubei Key Laboratory of Cardiology, Wuhan, Hubei 430060, P.R. China
}

Received February 12, 2018; Accepted November 1, 2018

DOI: $10.3892 /$ etm.2018.7000

\begin{abstract}
In peripheral arterial disease (PAD), angiogenesis is the major process involved in repairing the microvasculature in the ischemic lower limb. Curcumin, a monomer isolated from turmeric roots, has been demonstrated to have pro- and anti-angiogenic effects under different circumstances. Previous studies have indicated that curcumin treatment improves tissue repair and perfusion recovery in a mouse model of diabetic PAD. However, the effects of curcumin on PAD under non-diabetic conditions has remained elusive, In the present study, mice with PAD and a normal glycaemic profile were treated with curcumin, which improved perfusion recovery, increased capillary density and elevated microRNA (miR)-93 expression in ischemic muscle tissue. In cultured endothelial cells under simulated ischemia, curcumin improved endothelial cell viability and enhanced tube formation. However, following miR-93 knockdown using a microRNA inhibitor, endothelial cell tube formation was inhibited. Furthermore, in the presence of the miR-93 inhibitor, curcumin did not alter endothelial cell viability or tube formation. These results demonstrate that curcumin had beneficial effects in non-diabetic PAD by improving angiogenesis, which may have been achieved partially via the promotion of miR-93 expression.
\end{abstract}

\section{Introduction}

Peripheral arterial disease (PAD), caused by occlusion of the arteries extending to the lower extremities, is a growing medical problem that affects $>200$ million individuals worldwide (1-3). Delivery of oxygen, nutrients and other mediators to

Correspondence to: Dr Ronghua He, Department of Cardiology, The Central Hospital of Xiaogan, Wuhan University of Science and Technology, 6 Guangchang Road, Xiaogan, Hubei 432000, P.R. China

E-mail: ronghuahecn@sohu.com

Key words: curcumin, angiogenesis, peripheral arterial disease, microRNA-93, endothelial cells ischemic sites in patients with PAD via the blood circulation is dependent on neovascularization, including angiogenesis and arteriogenesis (4-7). However, at present, no medications are available to induce functional neovascularization and thereby treat patients with PAD (8-10).

Curcumin is a bright-yellow compound isolated from the root of Curcuma longa, which is a member of the ginger family, and has traditionally been used to treat a variety of clinical conditions, including cancer, Alzheimer's disease and insulin resistance (11-13). Previous studies suggested that curcumin induces therapeutic angiogenesis and improves hind limb perfusion recovery after surgical femoral artery ligation in diabetic mice (14). However, whether curcumin provides a therapeutic benefit in PAD without diabetes has remained elusive. Considering that a large proportion of patients with PAD do not have any accompanying diabetes mellitus $(2,15)$, the present study was performed in order to investigate the potential effects of curcumin on perfusion recovery in a non-diabetic mouse model of PAD, and to elucidate the mechanism of action of angiogenic microRNA.

\section{Materials and methods}

Murine hindlimb ischemia(HLI). Unilateral HLI was generated via surgical ligation and excision of the femoral artery to create an experimental PAD model, as described previously (16). In the present study, 32 male BALB/c mice (age, 14 weeks; weight, 20-25 g) were anesthetized with $3 \%$ isoflurane. Immediately after HLI, the mice were randomized into two groups ( $\mathrm{n}=16$ in each): In the control group, the mice received $300 \mu 1$ olive oil only, and in the curcumin group, the mice received 1,000 mg/kg curcumin (Sigma-Aldrich; Merck KGaA, Darmstadt, Germany) in $300 \mu$ l olive oil. All mice received treatment by gavage, once per day for two weeks.

All procedures of the present study followed the Guide for the Care and Use of Laboratory Animals published by the US National Institutes of Health (publication no. 85-23, revised 1996). The experimental protocol was approved by the Committee on Animal Experiments of Wuhan University School of Medicine (Wuhan, China). The BALB/c mice were obtained from the Experimental Animal Center of Wuhan University (Wuhan, China). Mice were housed in a specific pathogen-free laboratory environment at a temperature 

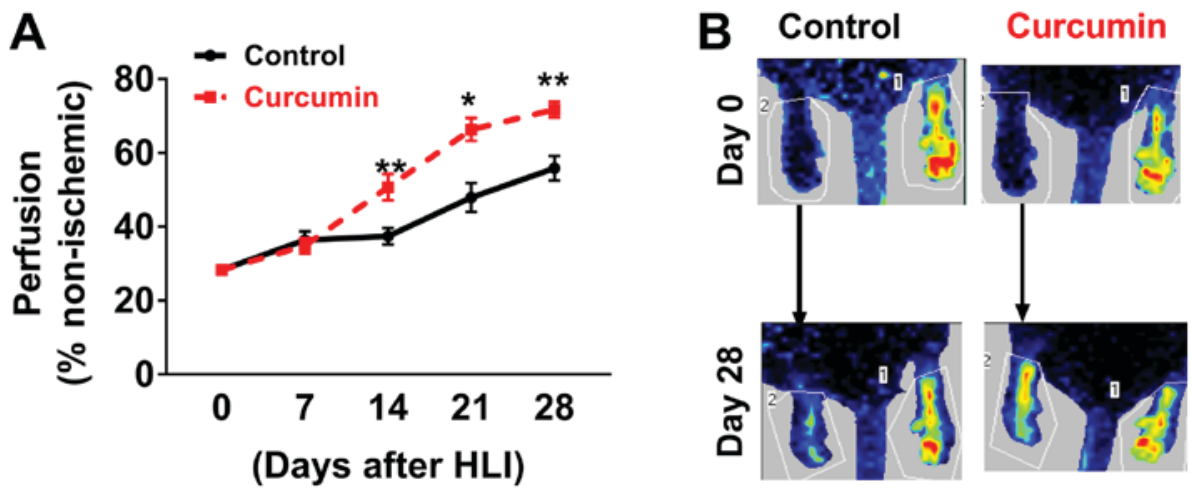

Figure 1. (A) LDPI revealed significantly increased perfusion recovery in BALB/c mice treated with curcumin at 14,21 and 28 days after HLI. (B) Representative LDPI profiles in mice on days 0 and 28 . Values are expressed as the mean \pm standard error of the mean (n=10/group). ${ }^{*} \mathrm{P}<0.05 ;{ }^{* * *} \mathrm{P}<0.01$ vs. control. LDPI, laser Doppler perfusion imaging; HLI, hind limb ischemia.

of $25^{\circ} \mathrm{C}$ and a constant humidity of $50 \pm 10 \%$ with free access to food and water under a 12-h light/dark cycle.

Perfusion recovery. Mice were anesthetized and subjected to a non-invasive assessment of ischemic and non-ischemic limb perfusion using a laser Doppler perfusion imaging system (LDPI; Perimed Instruments AB, Stockholm, Sweden) at 0, 7, 14, 21 and 28 days after HLI, as described previously (17). Perfusion of the ischemic limb was quantified and normalized to the non-surgical limb, and the results are presented as a percentage of the values in the non-ischemic side.

Immunofluorescence. Mice were sacrificed in a $\mathrm{CO}_{2}$ chamber at 28 days after HLI, and the gastrocnemius anterior muscles from the ischemic side were cryo-sectioned in $6-\mu \mathrm{m}$ sections. Anti-CD31 antibody (rat anti-mouse CD31; cat. no. 550274; 1:100 dilution; BD Pharmingen, San Jose, CA, USA) was applied to acetone-fixed sections (fixed for $-20^{\circ} \mathrm{C}$ for $10 \mathrm{~min}$ ) of ischemic gastrocnemius muscle tissue, followed by incubation overnight at $4^{\circ} \mathrm{C}$ with an Alexa Fluor 555 anti-rabbit secondary antibody (1:400 dilution; cat. no. BM2004; Boster Biological Technology, Wuhan, China). Images were acquired using an Olympus IX71 high-magnification microscope (Olympus, Tokyo, Japan). Capillary densities were analyzed by counting in four randomly selected high-power fields (magnification, x100) and expressed as the number of $\mathrm{CD} 31^{+}$cells per field.

$R N A$ isolation and reverse transcription-quantitative polymerase chain reaction (RT-qPCR) analysis. Total RNA was isolated from tissue or cells using a PureLink ${ }^{\circledR}$ RNA Mini kit (cat. no. 12183018A; Thermo Fisher Scientific, Inc., Waltham, MA, USA) according to the manufacturer's protocol. Real-time qPCR for microRNA (miR) quantification and a miR assay (assay no. 001090; cat. no: 4427975; Thermo Fisher Scientific, Inc.) were used for RT-qPCR according to the manufacturer's protocols. Small nucleolar RNA MBII-202 (assay no. 001095; Thermo Fisher Scientific, Inc.) served as an internal control for miR quantification. The quantification cycle $(\mathrm{Cq})$ value obtained for each gene was normalized to that of the respective internal control $(\Delta \mathrm{Cq})$. Each gene was then further normalized to the average $\Delta \mathrm{Cq}$ value of its control group $(\Delta \Delta \mathrm{Cq})$. The final fold expression changes were calculated using the $2^{-\Delta \Delta \mathrm{Cq}}$ equation (18).
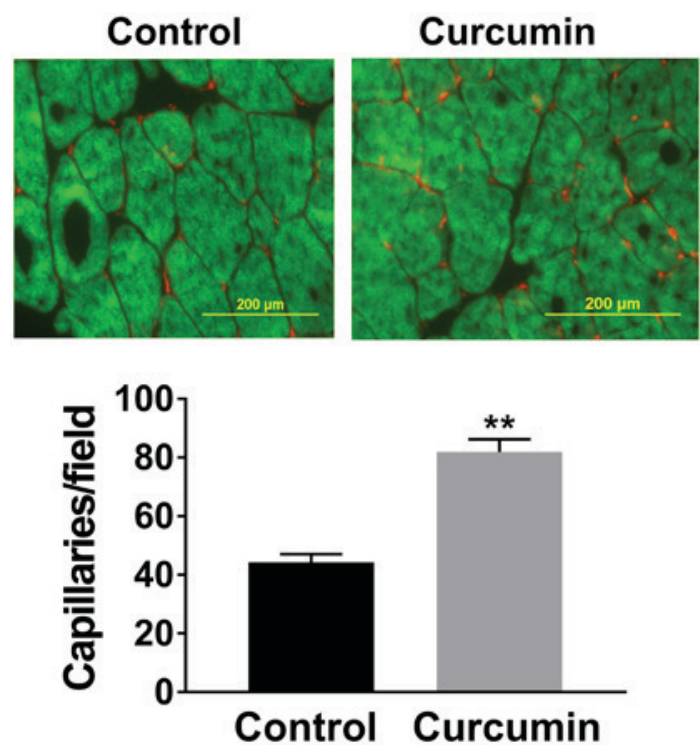

Figure 2. Curcumin treatment increased the capillary density in ischemic gastrocnemius muscle tissue at 28 days after hind limb ischemia, as compared with that in mice receiving vehicle (scale bar, $200 \mu \mathrm{M}$ ). Values are expressed as the mean \pm standard error of the mean ( $n=10 /$ group). Green staining represents skeletal muscle, red staining indicates capillaries in ischemic muscle tissue. ${ }^{* *} \mathrm{P}<0.01$ vs. control.

Cell culture and in vitro transfection. Human umbilical vein endothelial cells (HUVECs) were isolated from a donor umbilical cord, as described previously (19), and then cultured in endothelial cell growth medium (Cell Applications, Inc., San Diego, CA, USA) supplemented with $10 \%$ fetal bovine serum (Wuhan Boster Biological Technology). To mimic endothelial cells under ischemic conditions as a model for HLI, HUVECs were subjected to hypoxia ( $2 \%$ oxygen; BioSpherix, Lacona, NY, USA) and serum starvation (HSS). The use of HUVECs was approved by the Institutional Review Boards of Wuhan University (Wuhan, China).

In vitro transfection of miRNA inhibitors was used to knock down miR-93 expression in HUVECs, as described previously (20). In brief, a reverse transfection protocol using neofx transfection agent (Ambion, Austin, TX, USA) was used to transfect miR-93 inhibitor, or miRNA inhibitor negative control (cat. no. 4464084; Thermo Fisher Scientific, Inc) into HUVECs for $48 \mathrm{~h}$. 

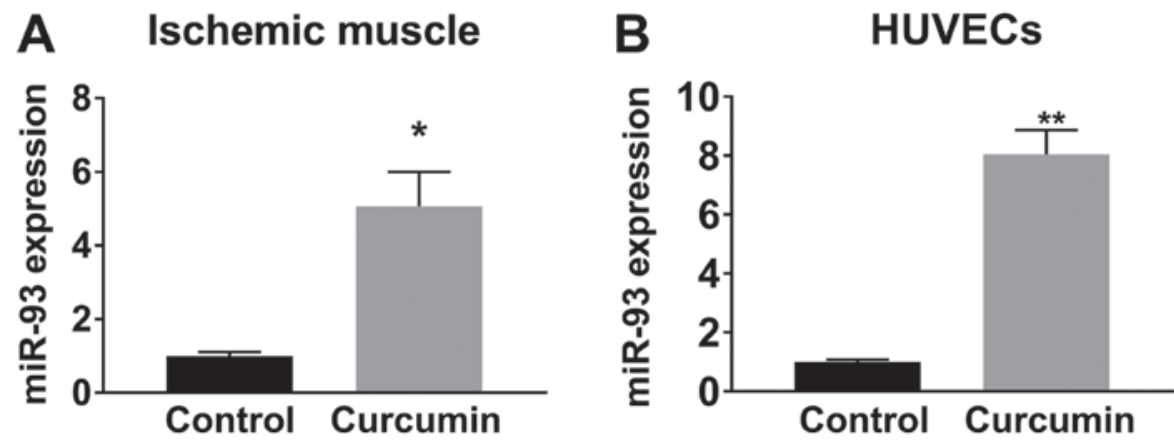

Figure 3. Curcumin treatment caused an upregulation in miR-93 expression (A) in ischemic muscle tissues ( $5.05 \pm 0.94$ fold) at 7 days after hind limb ischemia and (B) in HUVECs $(8.03 \pm 0.82$ fold) after $12 \mathrm{~h}$ under hypoxia and serum starvation conditions. Values are expressed as the mean \pm standard error of the mean ( $\mathrm{n}=10 /$ group) ${ }^{*} \mathrm{P}<0.05 ;{ }^{* *} \mathrm{P}<0.01$ vs. control. miR, microRNA; HUVECs, human umbilical cord vascular endothelial cells.

Cellular viability and angiogenesis assay. For assessment of cellular viability, HUVECs transfected with an miR-93 inhibitor or control miR were seeded into a 96-well plate at a density of $1 \times 10^{4}$ cells/well ( $\mathrm{n}=8 /$ group), and then cultured under HSS conditions with/without curcumin $(10 \mu \mathrm{M})$ for $48 \mathrm{~h}$. Subsequently, the cell viability was assessed using tetrazolium dye incorporation (BioVision, Milpitas, CA, USA).

In vitro angiogenesis assays were performed as previously described (20), under HSS conditions. In brief, HUVECs transfected with miR-93 inhibitor or control were seeded in 96-well dishes coated with growth factor-reduced Matrigel (BD Biosciences, Franklin Lakes, NJ, USA) at a density of $1 \times 10^{4}$ cells/well, and then exposed to HSS conditions in the presence of curcumin $(10 \mu \mathrm{M})$ or vehicle alone for $12 \mathrm{~h}$ to assess tube formation. Each set of conditions was replicated in 6 wells from the 96-well dish. The degree of tube formation was determined by measuring the length of the tubes and the number of loops in each well under a magnification, $\mathrm{x} 40$ using ImageJ software 1.15K (National Institutes of Health, Bethesda, MD, USA). Each experiment was repeated using at least two different batches of HUVECs in total.

Statistical analysis. Statistical analysis was performed with GraphPad Prism 7.0 software (GraphPad Inc., La Jolla, CA, USA). An unpaired t-test was used for comparisons between two groups; comparisons between $\geq 3$ groups were performed with one-way analysis of variance and Tukey's post-hoc test. $\mathrm{P}<0.05$ was considered to indicate a statistically significant difference.

\section{Results}

Curcumin improves perfusion recovery, angiogenesis and causes upregulation of miR-93 expression in experimental $P A D$. LDPI imaging revealed that BALB/c mice receiving curcumin experienced better perfusion recovery at 14, 21 and 28 days after HLI compared with those receiving olive oil (Fig. 1). Immunostaining with CD31 to visualize capillaries indicated that after 28 days of post-HLI treatment, the gastrocnemius anterior muscles from the ischemic side of the mice receiving curcumin exhibited a higher capillary density compared with those receiving olive oil ( $81.9 \pm 4.3$ vs. $44.3 \pm 2.7$ capillaries/field, n=10/group; $\mathrm{P}<0.01$; Fig. 2). A previous study indicated that miR-93 is a potent regulator of angiogenesis in the ischemic limbs of patients with PAD and in animal models (20). Given that curcumin induced angiogenesis as a therapeutic benefit after HLI, the level of miR-93 was assessed, revealing that curcumin treatment increased miR-93 expression by $\sim 5$ fold ( $\mathrm{n}=5 /$ group) in the ischemic muscle at 7 days after HLI (Fig. 3A).

Curcumin therapy increases angiogenesis under hypoxia. In HUVECs cultured under HSS conditions (mimicking in vivo ischemia), curcumin treatment for $12 \mathrm{~h}$ significantly increased miR-93 expression (Fig. 3B), consistent with the in vivo results obtained with ischemic muscle tissue. In addition, curcumin increased endothelial cell viability (Fig. 4A) and tube formation (Fig. 4B) in vitro under HSS conditions. It was also revealed that miR-93 knockdown using a miR-93 inhibitor reduced angiogenesis and curcumin-induced angiogenesis and endothelial cell survival were attenuated when miR-93 was knocked down by an miR-93 inhibitor in vitro (Fig. $4 \mathrm{~A}$ and $\mathrm{B}$ ).

\section{Discussion}

To the best of our knowledge, the present study is the first to demonstrate that curcumin improves angiogenesis and perfusion recovery in non-diabetic experimental PAD. Furthermore, it was indicated that curcumin treatment increased miR-93 expression in ischemic muscle tissue and cultured endothelial cells, and that miR-93 elevation may be involved in curcumin-induced therapeutic angiogenesis under ischemic conditions.

Previous studies have demonstrated that curcumin has a protective effect on ischemic limbs in diabetic mouse models $(14,15)$. However, the effects of curcumin on limb ischemia in non-diabetic subjects have remained to be assessed. Angiogenesis is an important process of new blood vessel formation, which includes the stimulation, promotion and stabilization of endothelial cells; it is a key factor in the perfusion recovery of tissue following ischemia. Curcumin has a pro-angiogenic effect on wound healing and HLI in type 1 diabetes (21). However, it has been indicated to have anti-angiogenic effects in pituitary adenomas and hepatic cancer $(11,22)$. Taken together, curcumin exhibits bi-directional effects under different disease conditions. Therefore, 
A

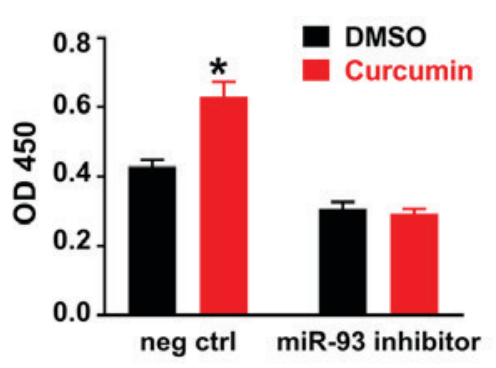

B

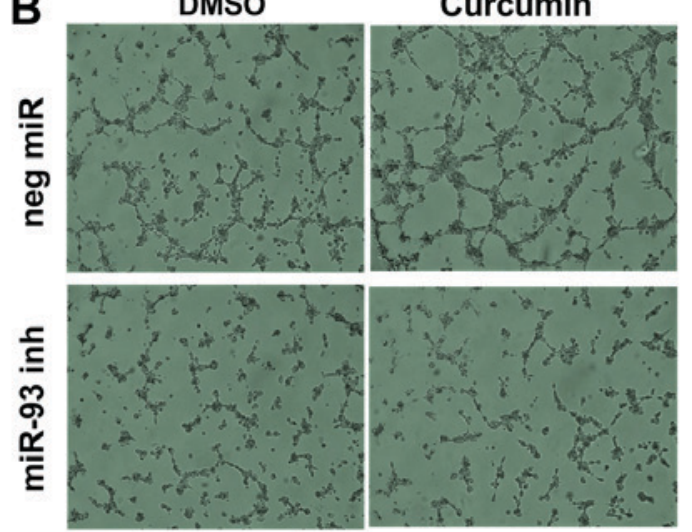

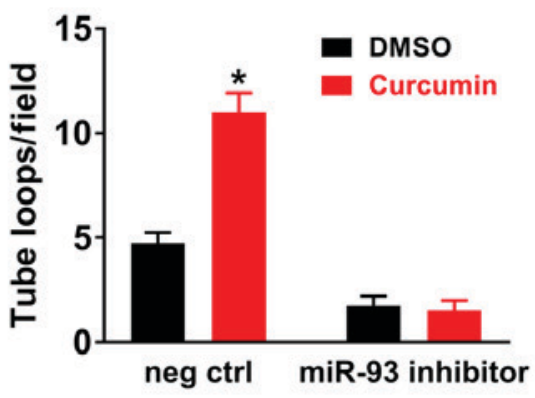

Figure 4. Curcumin treatment increases the cell viability and tube formation of cultured HUVECs under HSS conditions. (A) HUVECs were incubated with curcumin or DMSO for $48 \mathrm{~h}$, and curcumin significantly increased the cell viability. (B) HUVECs were seeded on 96-well plates coated with Matrigel; curcumin treatment for $12 \mathrm{~h}$ significantly increased tube formation, as indicated by increased loops (magnification, $\mathrm{x} 40$ ). DMSO (0.02\%, volume concentration in the medium) served as a negative control for curcumin, as curcumin used in the in vitro study was dissolved in DMSO. miR-93 knockdown reduces angiogenesis in vitro, furthermore following miR-93 knockdown, curcumin did not alter endothelial cell viability or tube formation. Values are expressed as the mean \pm standard error of the mean. "P<0.05 vs. control. HUVECs, human umbilical cord vascular endothelial cells; HSS, hypoxia and serum starvation; OD 450, optical density at $450 \mathrm{~nm}$; neg ctrl, negative control; miR, microRNA; inh, inhibitor; DMSO, dimethylsulfoxide.

under non-diabetic conditions, the effects of curcumin on angiogenesis in PAD require further study.

A noteworthy result of the present study is that curcumin improves perfusion recovery after HLI through the induction of miR-93 upregulation in ischemic endothelial cells. miRs are a group of small non-coding RNAs containing $\sim 22$ nucleotides that function through RNA silencing and the post-transcriptional regulation of gene expression (23-27). miR-93 has been reported to act as a potent mediator to induce neovascularization in PAD. In a mouse model of PAD, miR-93 knockdown was reported to reduce angiogenesis and perfusion recovery; conversely, miR-93 overexpression improved perfusion recovery and angiogenesis by targeting cell cycle regulatory pathways (21). A more recent study indicated that miR-93 induces macrophage M2 polarization, which eventually leads to enhanced angiogenesis and arteriogenesis in a mouse model of PAD (28). In the present study, treatment with curcumin was identified to cause an upregulation of miR-93 in ischemic muscle tissue and endothelial cells. In addition, miR-93 inhibition blocked curcumin-induced therapeutic angiogenesis in vitro. This may suggest that miR-93 is involved in the therapeutic effects of curcumin on PAD.

At present, limited therapies are available for PAD, and no known treatment is capable of increasing neovascularization in the ischemic limbs of patients with PAD $(10,17)$. Combined with the previous result that curcumin improves outcomes in diabetic PAD, the present study suggests that curcumin may serve as an effective alternative treatment approach for PAD in non-diabetic subjects.

\section{Acknowledgements}

Not applicable.

\section{Funding}

No funding was received.

\section{Availability of data and materials}

The datasets used and/or analyzed during the current study are available from the corresponding author on reasonable request.

\section{Authors' contributions}

JZ and RH conceived and designed the current study. JZ, QW and RH wrote and edited the manuscript. JZ, QW, GR, JQ and $\mathrm{RH}$ performed the experiments, and read and approved the final manuscript.

\section{Ethics approval and consent to participate}

The experimental animal protocol was approved by the Committee on Animal Experiments of Wuhan University School of Medicine. The use of HUVECs isolated from donor umbilical cords was approved by the Institutional Review Boards of Wuhan University.

\section{Patient consent for publication}

Not applicable.

\section{Competing interests}

The authors declare that they have no competing interests.

\section{References}

1. Guerchet M, Aboyans V, Mbelesso P, Mouanga AM, Salazar J, Bandzouzi B, Tabo A, Clément JP, Preux PM and Lacroix P: Epidemiology of peripheral artery disease in elder general population of two cities of Central Africa: Bangui and Brazzaville. Eur J Vasc Endovasc Surg 44: 164-169, 2012.

2. Criqui $\mathrm{MH}$ and Aboyans V: Epidemiology of peripheral artery disease. Circ Res 116: 1509-1526, 2015.

3. Fowkes FG, Aboyans V, Fowkes FJ, McDermott MM, Sampson UK and Criqui MH: Peripheral artery disease: Epidemiology and global perspectives. Nat Rev Cardiol 14: 156-170, 2017. 
4. Espinola-Klein C and Savvidis S: Peripheral arterial disease. Epidemiology, symptoms and diagnosis. Internist (Berl) 50 919-926, 2009 (In German).

5. Grundmann S, Piek JJ, Pasterkamp G and Hoefer IE: Arteriogenesis: Basic mechanisms and therapeutic stimulation. Eur J Clin Invest 37: 755-766, 2007.

6. Hirsch AT: Treatment of peripheral arterial disease-extending 'intervention' to 'therapeutic choice'. N Engl J Med 354: 1944-1947, 2006.

7. Joh JH, Joo SH and Park HC: Simultaneous hybrid revascularization for symptomatic lower extremity arterial occlusive disease. Exp Ther Med 7: 804-810, 2014.

8. Aviles RJ, Annex BH and Lederman RJ: Testing clinical therapeutic angiogenesis using basic fibroblast growth factor (FGF-2). Brit J Pharmacol 140: 637-646, 2003.

9. Owens CD and Conte MS: Medical management of peripheral arterial disease: Bridging the 'Gap'? Circulation 126: 1319-1321, 2012.

10. Annex BH: Therapeutic angiogenesis for critical limb ischaemia. Nat Rev Cardiol 10: 387-396, 2013.

11. Allegra A, Innao V, Russo S, Gerace D, Alonci A and Musolino C: Anticancer activity of curcumin and its analogues: Preclinical and clinical studies. Cancer Invest 35: 1-22, 2017.

12. Goozee KG, Shah TM, Sohrabi HR, Rainey-Smith SR, Brown B, Verdile $G$ and Martins RN: Examining the potential clinical value of curcumin in the prevention and diagnosis of Alzheimer's disease. Br J Nutr 115: 449-465, 2016.

13. Jiménez-Osorio AS, Monroy A and Alavez S: Curcumin and insulin resistance-Molecular targets and clinical evidences. Biofactors 42: 561-580, 2016

14. You J, Sun J, Ma T, Yang Z, Wang X, Zhang Z, Li J, Wang L, Ii M, Yang J and Shen Z: Curcumin induces therapeutic angiogenesis in a diabetic mouse hindlimb ischemia model via modulating the function of endothelial progenitor cells. Stem Cell Res Ther 8: 182, 2017.

15. Barrett PM, Wall CAM and Stack AG: Peripheral artery disease prevalence and mortality trends of United States dialysis population: 1995-2005. Irish J Med Sci 179: S409-S410, 2010

16. Waters RE, Terjung RL, Peters KG and Annex BH: Preclinical models of human peripheral arterial occlusive disease: Implications for investigation of therapeutic agents. J Appl Physiol (1985) 97: 773-780, 2004.

17. Albadawi H, Oklu R, Cormier NR, O'Keefe RM, Heaton JT, Kobler JB, Austen WG and Watkins MT: Hind limb ischemia-reperfusion injury in diet-induced obese mice. J Surg Res 190: 683-691, 2014.
18. Livak KJ and Schmittgen TD: Analysis of relative gene expression data using real-time quantitative PCR and the 2(-Delta Delta C(T)) method. Methods 25: 402-408, 2001.

19. Baudin B, Bruneel A, Bosselut N and Vaubourdolle M: A protocol for isolation and culture of human umbilical vein endothelial cells. Nat Protoc 2: 481-485, 2007.

20. Hazarika S, Farber CR, Dokun AO, Pitsillides AN, Wang T, Lye RJ and Annex BH: MicroRNA-93 controls perfusion recovery after hindlimb ischemia by modulating expression of multiple genes in the cell cycle pathway. Circulation 127: 1818-1828, 2013.

21. Elavarasu S, Suthanthiran T, Thangavelu A, Alex S, Palanisamy VK and Kumar TS: Evaluation of superoxide dismutase levels in local drug delivery system containing $0.2 \%$ curcumin strip as an adjunct to scaling and root planing in chronic periodontitis: A clinical and biochemical study. J Pharm Bioallied Sci 8 (Suppl 1): S48-S52, 2016.

22. Huang F, Yao Y, Wu J, Liu Q, Zhang J, Pu X, Zhang Q and Xia L: Curcumin inhibits gastric cancer-derived mesenchymal stem cells mediated angiogenesis by regulating NF-kB/VEGF signaling. Am J Transl Res 9: 5538-5547, 2017.

23. He L and Hannon GJ: MicroRNAs: Small RNAs with a big role in gene regulation. Nat Rev Genet 5: 522-531, 2004.

24. González-Duarte RJ, Cázares-Ordoñez V and Ávila-Chávez E: The microRNA biogenesis machinery: Regulation by steroid hormones and alterations in cancer. Rev Invest Clin 66: 460-464, 2014.

25. Ha M and Kim VN: Regulation of microRNA biogenesis. Nat Rev Mol Cell Biol 15: 509-524, 2014.

26. Jonas S and Izaurralde E: Towards a molecular understanding of microRNA-mediated gene silencing. Nat Rev Genet 16: 421-433, 2015.

27. Zhou X, Yuan P and He Y: Role of microRNAs in peripheral artery disease (review). Mol Med Rep 6: 695-700, 2012.

28. Ganta VC, Choi MH, Kutateladze A, Fox TE, Farber CR and Annex BH: A MicroRNA93-interferon regulatory factor-9-immunoresponsive gene-1-itaconic acid pathway modulatesM2-like macrophage polarization to revascularize ischemic muscle. Circulation 135: 2403-2425, 2017.

This work is licensed under a Creative Commons Attribution-NonCommercial-NoDerivatives 4.0 International (CC BY-NC-ND 4.0) License. 